\title{
Profound mental retardation, characteristic facies with midfacial hypoplasia and premature frontotemporal balding, muscular hypotrophy, and small patellae in two unrelated male patients
}

\author{
J-P Fryns, P Thiry, J Geutjens, E Smeets, L Vinken, H Van den Berghe
}

\begin{abstract}
Two profoundly mentally retarded, unrelated males are reported with an unidentified multiple congenital anomaly/ mental retardation syndrome, including early balding, patella luxations, small hands and feet, and hypogonadism, similar to a previous publication in this journal of a severely mentally retarded male patient with dysmorphic features. (f Med Genet 1993;30:319-21)
\end{abstract}

\section{Case reports \\ PATIENT 1}

Patient 1 , a male, is now 36 years old. He was born after a normal term pregnancy (birth weight $3400 \mathrm{~g}$ ) and was the fifth of six children, one girl and five boys. The parents were healthy and unrelated and the family history was unremarkable. At his birth the mother was 38 and the father 42 years old. Psychomotor retardation was noted from the beginning and feeding was difficult. At the age of 5 months he was admitted to a paediatric hospital because of recurrent respiratory infections. He only started to walk a few steps independently after the age of 3 years. Speech never developed and from the age of 7 years he started to mutilate himself. Generalised epileptic seizures were noted between the ages of 15 and 21 years, but he is now no longer epileptic and antiepileptic medication has been stopped. He was admitted to a school for the severely mentally retarded at a young age and to an institute for the severely retarded at the age of 21 years. Clinical examination has remained almost unchanged over the last 15 years. He is profoundly mentally retarded (mental age below 10 months) and is unable to speak or feed himself. $\mathrm{He}$ is able to walk independently over a short distance. He is anxious, sometimes aggressive, and mutilates himself. Height is $162 \mathrm{~cm}$, span $165 \mathrm{~cm}$, weight $44 \mathrm{~kg}$, and head circumference $54 \mathrm{~cm}$. He shows distinct craniofacial dysmorphism (fig 1) with frontotemporal balding, a high forehead, deep set eyes, blue irides, a bulbous, bifid nasal tip, and a large mouth with thin, everted upper lips. The hands and feet are small with a large gap between the first and second toes. Since admission to the institute he has shown marked general hypotrophy (height $162 \mathrm{~cm}$, weight $44 \mathrm{~kg}$ ) with poor muscle development and almost no subcutaneous fat.
Both patellae are small $(2 / 2.5 \mathrm{~cm})$ and laterally placed. There is a $15^{\circ}$ thoracolumbar scoliosis and neurological examination showed an unsteady gait, generalised hypotonia, and a mild pyramidal syndrome. Secondary sexual development is normal with normal testes (2/ $3 \mathrm{~cm}$ ).

Routine haematological and biochemical screening was normal. Chromosomal analysis on two different lymphocyte cultures showed a normal 46,XY male karyotype after $G$ banding. Fragile $X$ screening was negative (100 cells of an M199 culture).

Non-specific changes were noted on different EEGs and CT scan of the brain showed a broad ventricular system with large lateral ventricles. Because of the marked general hypotrophy with poor muscle development further neuromuscular examinations were performed when he was admitted at the age of 22 years. Serum creatine kinase was slightly raised with increased $M M$ isozymes (106 U/l, normal 0 to $65 \mathrm{U} / 1$ ); electromyography showed a pattern of short duration, small amplitude, polyphasic potentials. Nerve conduction velocities were normal. Muscle biopsy of the right gastrocnemius showed dystrophic changes with variation in fibre size (many atrophic and some hypertrophic fibres), internal displacement of nuclei, split fibres, necrosis, regeneration, and endomyosial fibrosis. Over the next 14 years no clinical progression was noted and now, at the age of 36 years, neuromotor functioning is identical to that at the age of 21 years. Neuromuscular reinvestigations were refused by the family.

\section{PATIENT 2}

Patient 2, a male, is now 37 years old. He was born after an uncomplicated pregnancy. Delivery was normal. He is the fourth child of six (one girl and five boys) of healthy, non-consanguineous parents. Family history is negative with regard to severe mental handicap and congenital malformations. At his birth the mother was 26 and the father 29 years old. Severe psychomotor retardation was noted from the beginning and he was bedridden for the following years. At the age of 7 years he learned to walk with support and to crawl. $\mathrm{He}$ was epileptic with tonic-clonic seizures until the age of 28 years but is now no longer epileptic. He is profoundly mentally retarded (mental age below 10 months) and was admit- 


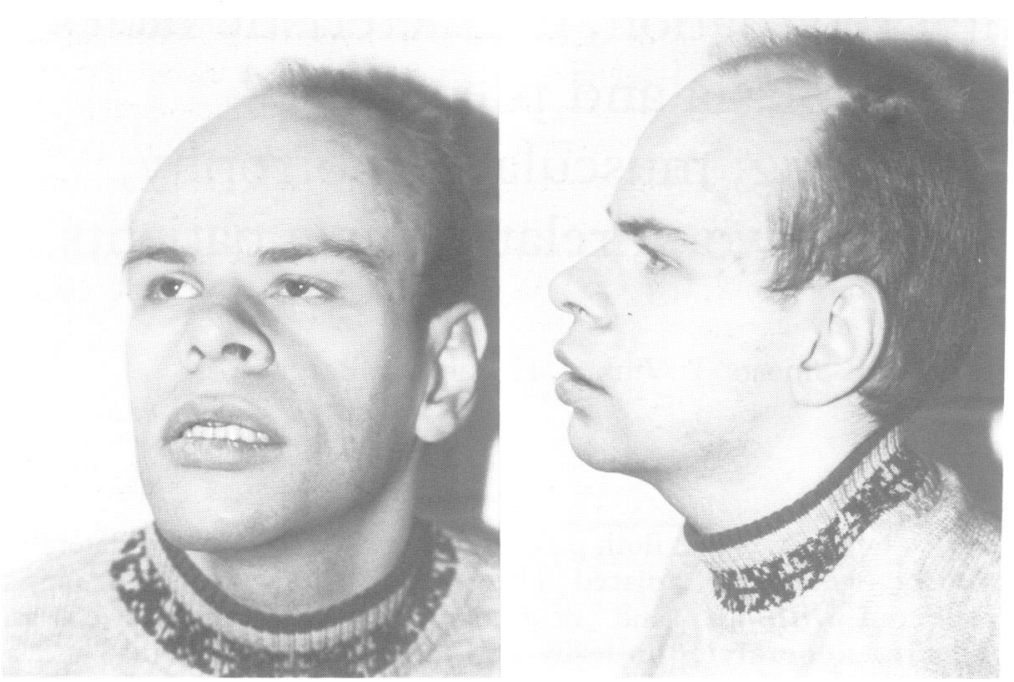

Figure 1 The craniofacial dysmorphism in patient 1.

ted to an institute for severely mentally retarded adult patients at the age of 24 years.

Height is $154 \mathrm{~cm}$, span $160 \mathrm{~cm}$, weight $36 \mathrm{~kg}$, and head circumference $52 \mathrm{~cm}$. Since his admission clinical findings and motor function have remained unchanged.

Craniofacial dysmorphism (fig 2 ) is obvious with a high, bulging forehead, frontotemporal balding which became more evident after the age of 25 years, deep set eyes, blue irides, bulbous, bifid nasal tip, and a large mouth with a thin, everted upper lip. There is bilateral keratoconus and convergent strabismus of the left eye. The hands and feet are small and slender with short toes and bilateral metatarsus adductus. There is marked general hypotrophy, little subcutaneous fat, poor muscle development, thin and distally hypotrophic legs, thoracic hyperkyphosis, and a $20^{\circ}$ thoracolumbar scoliosis. The patellae are small (2/ $1 \mathrm{~cm}$ ) and laterally placed.

Neurological examination showed spastic paraparesis of the lower legs, tremor, dysmetria, and positive pseudobulbar reflexes. Secondary sexual development was normal but
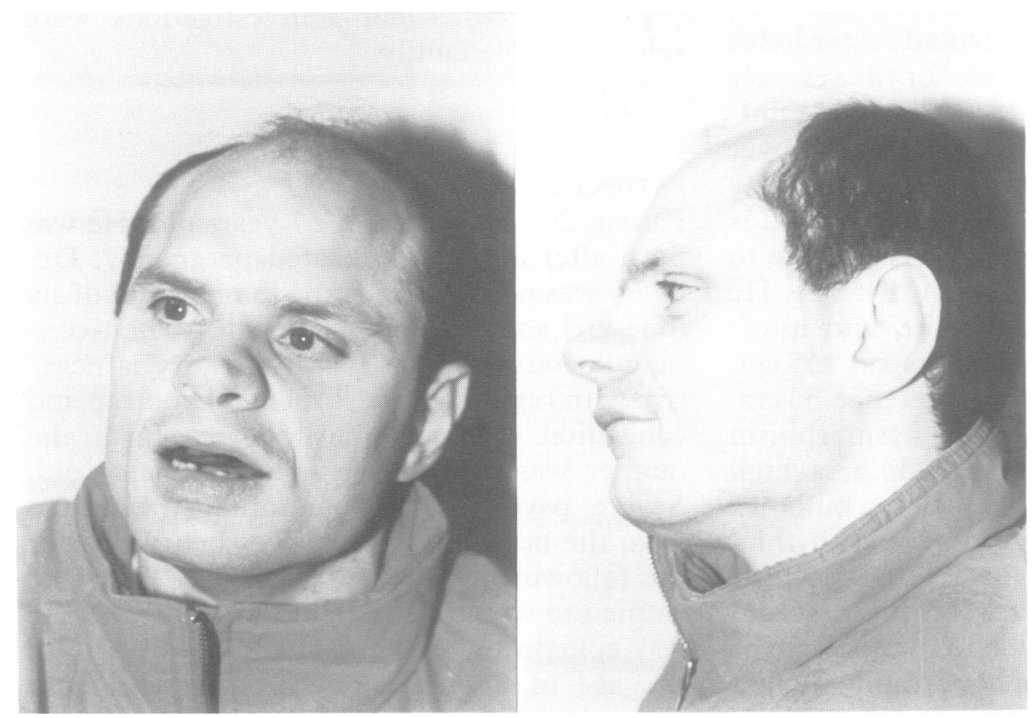

Figure 2 Identical dysmorphism in patient 2 both testes are small $(1 / 2 \mathrm{~cm})$. Routine haematological and metabolic screening was normal.

Chromosome analysis on a peripheral blood lymphocyte culture showed a normal $46, \mathrm{XY}$ male karyotype. Fragile $\mathrm{X}$ screening was negative (100 cells of an M199 culture).

CT scan of the brain showed marked general cerebellar atrophy and large lateral ventricles. Electroencephalography and electromyography were normal. Creatine kinase levels were normal. Muscle biopsy (right gastrocnemius) showed a fibre type disproportion and generalised small fibres but no dystrophic changes.

\section{Discussion}

These two unrelated, profoundly mentally retarded males were examined in the early 1980s during a systematic genetic-aetiological study of the severely mentally handicapped. Great similarity in clinical symptoms and signs was noted in both patients from the beginning: normal pre- and perinatal history, profound mental handicap, slow motor development limited to walking with support over short distances after the age of 5 years, characteristic craniofacial dysmorphism, and generalised hypotrophy. The craniofacial dysmorphism with broad and high forehead, early frontotemporal balding, deep set, blue eyes, broad and bifid nasal tip, large mouth with short philtrum, and everted, thin upper lips was so similar that several medical and non-medical professionals thought they were brothers. In addition to the craniofacial dysmorphism there was marked general hypotrophy and poor muscle development. The muscle hypotrophy also involved the shoulder and limb girdles but was most pronounced in the distal parts of the lower legs which were thin and slender. The hands and feet were small. The patellae were hypoplastic and laterally placed. Both males have shown generalised epileptic fits from an early age but the epilepsy was well controlled after admission to the institute and antiepileptic medication was stopped some years ago. Neurological examination in both males showed hypotonia at rest and moderate signs of a pyramidal syndrome. Because of the poor muscle development further neuromuscular investigations were performed. In the first patient creatine kinase levels were slightly raised, electromyographic examination suggested a myopathic process, and dystrophic changes were found on muscle biopsy. In the second patient, however, creatine kinase levels and electromyography were normal, whereas the muscle biopsy showed fibre type disproportion and generalised small fibres without dystrophic changes. The clinical symptoms and motor function of the two patients has remained unchanged over the past 12 years and there is no clinical evidence of regression of locomotor abilities. With the available data we have no answer at present as to whether the morphological changes in the muscle biopsies are specific findings and whether they are related to the severe MCA/MR syndrome present in both males.

Recently, Scholte et $a l^{1}$ reported a severely 
mentally retarded male with early balding and facial dysmorphism identical to that seen in the present two males. In their patient the authors noted in addition patella luxations, acromicria, and hypogonadism. Small testes were present in the second patient of this report. Both had notably small, laterally placed patellae in addition to marked muscular hypotrophy. It seems probable that the patella luxations in the patient of Scholte $e t a l^{1}$ are secondary to the peripheral muscular hypotrophy which was apparently also present in their patient.

In conclusion, the two males described in this report and the patient reported by Scholte et $a l^{1}$ present a distinct $\mathrm{MCA} / \mathrm{MR}$ syndrome with profound mental retardation, characteristic face, general hypotrophy with almost complete absence of subcutaneous fat, and muscular hypoplasia. The pathological muscle changes observed in the present two patients may indicate that symptoms such as acromicria, thin and slender extremities, small or (sub)luxated patellae may be secondary to a myopathic process of still undefined nature and origin.

1 Scholte FA, Begeer JH, Van Essen AJ. Unknown syndrome: mental retardation with dysmorphic features, early balding, patella luxations, acromicria, and hypogonadism. $\mathfrak{f}$ Med Genet 1991;28:140-2. 